\title{
4SC-202 as a Potential Treatment for the Pediatric Brain Tumor Medulloblastoma
}

\author{
Shanta M. Messerli ${ }^{1, *}$, Mariah M. Hoffman ${ }^{2}$, Etienne Z. Gnimpieba ${ }^{2}$ (D), Hella Kohlhof ${ }^{3,4}$ \\ and Ratan D. Bhardwaj 1 \\ 1 Sanford Children's Health Research Center, Department of Pediatrics, Cancer Biology and \\ Immunotherapies, Sanford Research, Sanford School of Medicine, University of South Dakota, \\ Vermillion, SD 57069, USA; ratan.bhardwaj@gmail.com \\ 2 Biomedical Engineering Department, University of South Dakota, Vermillion, SD 57069, USA; \\ Mariah.Hoffman@coyotes.usd.edu (M.M.H.); Etienne.Gnimpieba@usd.edu (E.Z.G.) \\ 3 4SC AG, Fraunhoferstraße 22, 82152 Planegg, Germany; hella.kohlhof@immunic.de \\ 4 Immunic AG, Am Klopferspitz 19, 82152 Planegg, Germany \\ * Correspondence: Shanta.Messerli@sanfordhealth.org; Tel.: +1-508-364-1181
}

Received: 31 August 2017; Accepted: 23 October 2017; Published: 3 November 2017

\begin{abstract}
This project involves an examination of the effect of the small molecule inhibitor 4SC-202 on the growth of the pediatric brain cancer medulloblastoma. The small molecule inhibitor 4SC-202 significantly inhibits the viability of the pediatric desmoplastic cerebellar human medulloblastoma cell line DAOY, with an $\mathrm{IC}_{50}=58.1 \mathrm{nM}$, but does not affect the viability of noncancerous neural stem cells (NSC). 4SC-202 exposure inhibits hedgehog expression in the DAOY cell line. Furthermore, microarray analysis of human medulloblastoma patient tumors indicate significant upregulation of key targets in the Hedgehog signaling pathway and Protein Tyrosine Kinase (PTK7).
\end{abstract}

Keywords: 4SC-202; medulloblastoma; pediatric brain tumor; microarray; bioinformatics

\section{Introduction}

Medulloblastomas (MBs) and astrocytomas are some of the brain tumors most frequently found in children [1]. However, there are few effectual treatments and surgery on children is not effective as the removal of the entire brain tumor is difficult. Recently, transcriptome profiling of malignancies of the central nervous system (CNS) have provided important insights into potential targets for therapies [2-4]. Here, we examine how transcriptome profiling of pediatric medulloblastomas reveals targets for potential therapies.

In addition, we demonstrate how a novel orally available benzamide-type HDAC inhibitor, termed 4SC-202, significantly reduces the viability of medulloblastoma in culture, and lay the groundwork for potential preclinical work by using 4SC-202 in preclinical models of pediatric medulloblastoma. 4SC-202 specifically targets class I HDACs-HDAC1, HDAC2, and HDAC3- and the histone demethylase LSD1 (4SC company data: B.P.S. Bioscience Assay Report, Reaction Biology Corporation Assay Results, detailed results available upon request) [5]. Prior studies have demonstrated that 4SC-202 has strong anti-tumor activities in a number of cancer cell lines and preclinical models. For example, 4SC-202 has been demonstrated to reduce proliferation of all epithelial and mesenchymal urothelial carcinoma (UC) cell lines [6,7]. In addition, 4SC-202 has been shown to reduce proliferation and survival of human colorectal cells and inhibit growth of colorectal tumors in vivo [8]. 4SC-202 has been evaluated in a Phase I clinical trial for blood cancer, and anti-tumor efficacy has thus far been observed [9]. Based on these past studies and ongoing trials, the goal of this project was to examine the efficacy of 4SC-202 in pediatric medulloblastoma. 


\section{Materials and Methods}

\subsection{Cell Culture, Cell Viability Assays, and Immunocytochemistry}

Frozen adherent desmoplastic cerebellar medulloblastoma brain cells obtained from a four-year-old Caucasian male (DAOY; ATCC-HTB-186) were cultured in DMEM with $10 \%$ FBS, Penicillin at $37^{\circ} \mathrm{C}$. To test the effect of 4SC-202 on non-cancerous cells, the following control cell line was used: neural stem cells (MTI-GlobalStem) grown in Gibco Astroycte media containing DMEM, with N-2 supplement, and One Shot FBS. Cell viability assays were conducted with Cell Titer Glo 2.0 (Promega, Madison, WI, USA) using the Glowmax 96 microplate luminometer (Promega). One thousand cells were plated per 96 well in a Corning 96 Well Solid White Flat Bottom Polystyrene TC-Treated Microplates (Cat \#3917). Concentrations of 4 SC-202 ranging from $0.001 \mu \mathrm{M}-10 \mu \mathrm{M}$ were applied to DAOY cells for $72 \mathrm{~h}$. Control treatments consisted of DMSO at $0.001 \%$. Cell Viability was measured using Cell Titer Glo 2.0 (Promega, Madison, WI, USA), and luminescence was measured using the Glowmax software on a Glowmax 96 microplate luminometer (Promega, Madison, WI, USA).

For immunocytochemistry, DAOY medulloblastoma American Type Culture Collection (ATCC; Manassas, VA) cells were plated on PureCol purified collagen I (Biocare Medical, Pacheco, CA, USA) imaging dishes as described [10]. One week after plating, cells were treated with 4SC-202 at $58 \mathrm{nM}$ for 72 h, with control-treated cells treated with vehicle DMSO at $0.00001 \%$. Following 72 h exposure to 4SC-202, cells were fixed with $4 \%$ paraformaldehyde for $10 \mathrm{~min}$, washed $3 \times$ with $1 \times$ PBS, permeabilized with the $0.1 \%$ Triton- $X$ for 10 min, washed $3 \times$ with $1 \times$ PBS, blocked, and stained with primary antibody against Sonic Hedgehog conjugated to Alex Fluor 488 (EP1190Y, ab203961, Abcam, Cambridge, MA, USA) diluted 1:200, and secondary antibody Goat anti-rabbit IgG with Alexa Flour 488. Cells were imaged on a confocal Olympus FV1200 Laser Scanning Microscope.

\subsection{Spheroid Culture}

One thousand DAOY cells were plated in Corning ${ }^{\circledR}$ spheroid microplates and allowed to form spheroids for one week prior to being treated with $58 \mathrm{~nm} 4$ SC-202. After $72 \mathrm{~h}$ of treatment the spheroids were stained with caspase-3/7 (Cell Event Caspase-3/7 Green detection, Thermofisher, Waltham, MA, USA) and the NucRedDead 647 Ready Probe Reagent (Thermofisher, Waltham, MA, USA) and imaged using the OlympusIX71 microscope.

\subsection{Microarray Analysis}

Normalized mRNA expression values from Affymetrix HG-U133plus2 chips were extracted from the Gene Expression Omnibus (GEO) repository, dataset numbers GSE66354 and GSE35493 [11,12]. For GSE66354, gene expression data from eight SHH MBs, two normal cerebellums, three normal frontal lobes, one normal medulla, one normal midbrain, two normal occipital lobes, two normal parietal lobes, one normal temporal lobe, and one thalamus ( $n=13$ normal brain samples) were used in the analysis. For GSE35493, gene expression data from 17 MBs, 2 normal cerebellums, 1 normal frontal lobe, 2 normal temporal lobes, 2 normal occipital lobes, and 2 normal parietal lobes ( $\mathrm{n}=9$ normal brain samples) were used. Each gene of interest in these datasets was identified by a single Affymetrix probe set ID except EZH2 and HDAC2, which are each identified by two probe set IDs. EZH2 gene expression was determined based on the results from probe 203358_s_at and HDAC2 from probe 201833_at. Error is represented as the standard error of the mean (SEM). Significance was determined using Linear Models for Microarray Data (LIMMA) package in R Bioconductor through GEO2R between MB and normal cerebellum as well as between $\mathrm{MB}$ and all normal brain samples [13]. $P$ values were adjusted using the Benjamini and Hochberg procedure [14]. 


\section{Results}

\subsection{SC-202 Is Cytotoxic to Medulloblastoma in Cell Culture}

Exposure of the medulloblastoma cell line to 4SC-202 for $72 \mathrm{~h}$ significantly reduces viability at concentrations ranging from $0.001-10 \mu \mathrm{M}$ (Figure 1), but does not significantly affect the growth of the control NSCs. Concentrations ranging from $0.001-0.3 \mu \mathrm{M}$ were significantly different from control-treated cells, with a paired two-tailed $t$ test, $p<0.05$. Concentrations ranging from $1-10 \mu \mathrm{M}$ were significantly different from control-treated cells, with a paired two-tailed $t$ test, $p<0.001$.

To examine the mechanism of cell death, DAOY cells and spheroids were stained with caspase-3/7, Dead Red, and Hoechst 33342 for 72 hrs. following 4SC-202 exposure. There is an increase in Dead Red staining in the 4SC-202-treated cells relative to the control sample. Additionally, the caspase-3/7 stain is colocalized with Dead Red in drug-treated cells (Figure 2).

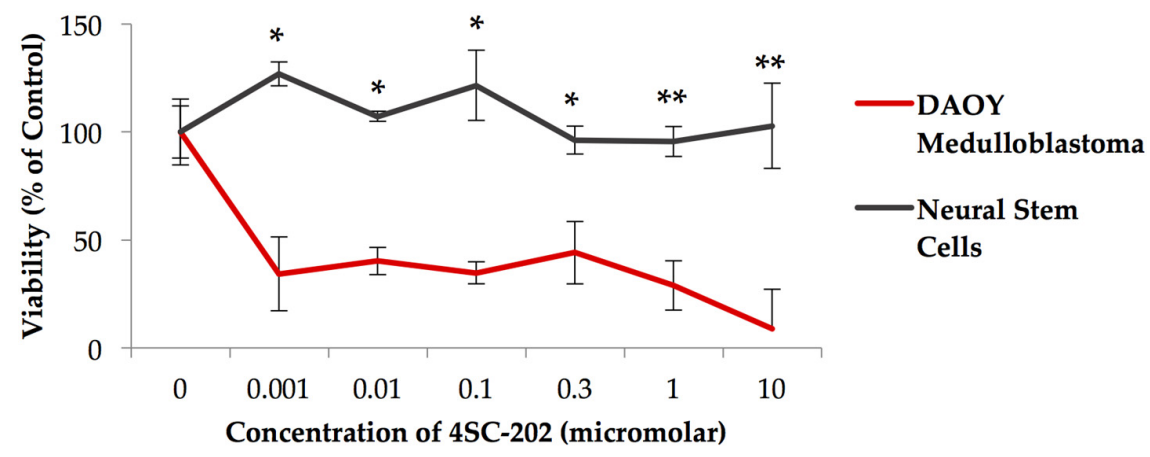

Figure 1. Exposure of DAOY medulloblastoma to 4SC-202 significantly reduces cell viability but does not affect the viability of control neural stem cells, with $p$ value $<0.01$ at concentrations ranging from $1-10 \mu \mathrm{M}\left({ }^{* *}\right)$, and $p$ value $<0.05\left(^{*}\right)$ at concentrations ranging from $0.001-1 \mu \mathrm{M}$. Viability was measured using Cell Titer Glo 2.0 (Promega).

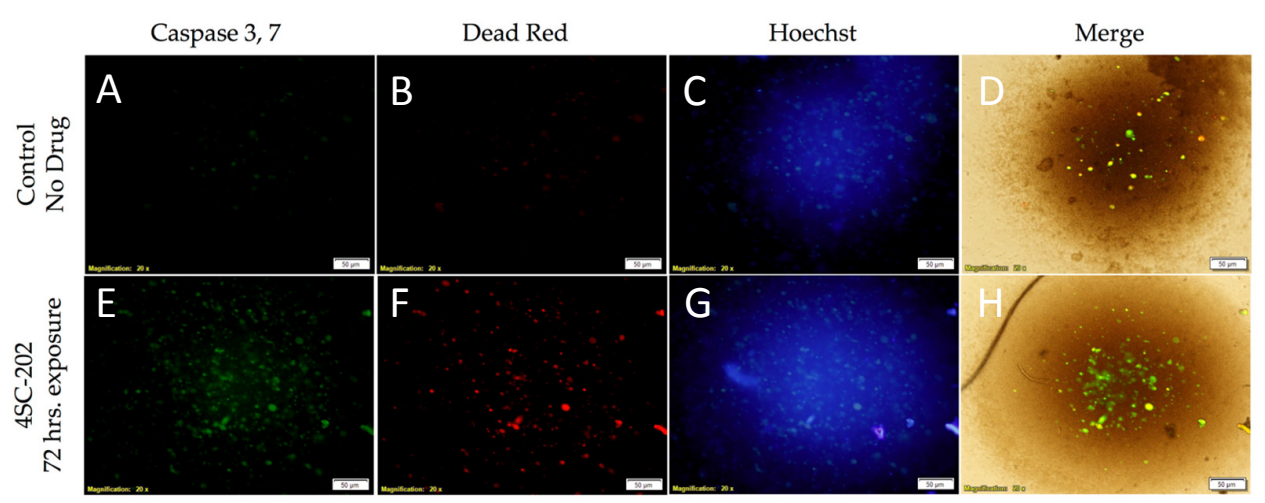

Figure 2. 4SC-202 induces caspase-3/7 activities in DAOY spheroids. DAOY spheroids were treated with 4SC-202 for $72 \mathrm{~h}$. Prior to being stained with caspase-3/7, Dead Red, and Hoechst stains. Caspase-3/7 activities are present in spheroids treated with 4SC-202 (E) but absent in DMSO control-treated spheroids (A). Increased dead cells were observed in the 4SC-202-treated spheroid (F) compared to DMSO control-treated spheroids (B). Hoechst stain visualizes nuclei in both conditions $(\mathbf{C}, \mathbf{G})$. Increased colocalization of caspase-3/7 with dead cells is visualized in (H) as compared to (D). Scale bar $=50$ microns.

\subsection{SC-202 Inhibits Hedgehog Immunoreactivity}

Exposure of the medulloblastoma cell line DAOY to $58 \mathrm{nM} 4 \mathrm{SC}-202$ blocks immunoreactivity for hedgehog. Positive staining for hedgehog was observed in control-treated DAOY cells (Figure 3B), 
which was reduced in 4SC-202 treated cells (Figure 3E). Reduced cell number and nuclei observed was also observed in 4SC-202 cells (Figure 3D) as compared to control-treated cells (Figure 3A).

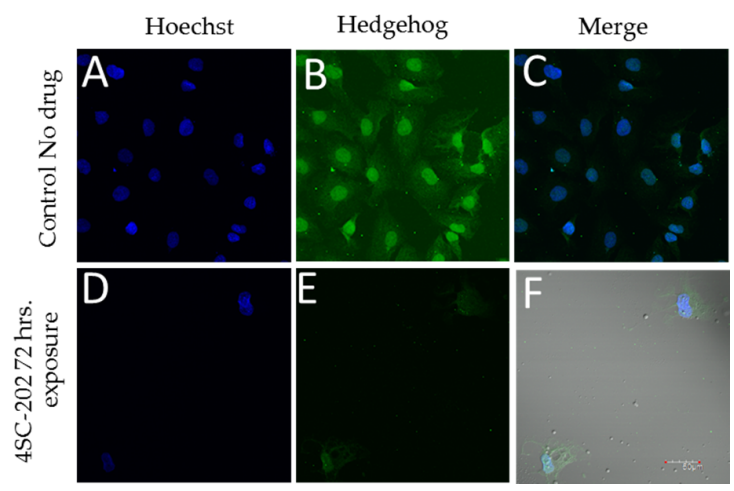

Figure 3. 4SC-202 reduces Hedgehog expression in DAOY medulloblastoma. Hedgehog immunoreactivity is present in control $(0.001 \%$ DMSO) (B) treated DAOY but significantly reduced in DAOY treated with 4SC-202 for $72 \mathrm{~h}$. (E, white arrows). The number of nuclei was significantly reduced following 4SC-202 exposure due to the cytotoxicity of 4SC-202 as assessed by staining with Hoechst dye (D) when compared to the number of nuclei in control treated DAOY (A). Colocalization of Hoechst and Hedgehog immunoreactivity is illustrated in control treated DAOY (C) and 4SC-202 treated DAOY (F), showing fewer hedgehog immunoreactivity treatment following 4SC-202 treatment. Scale bar $=50$ microns.

\subsection{Microarray Analysis of Human Medulloblastoma Tumors}

Microarray analysis from human medulloblastoma patients indicates significant upregulation of the gene expression levels in medulloblastoma for a number of known target proteins for 4SC-202 and SHH pathway proteins (Figure 4). LSD1, HDAC2, and HDAC3 are upregulated in both SHH-MBs and the nonspecific MB microarray results, but there is little evidence to suggest that $H D A C 1$ is upregulated. SHH mediators GLI2 and GLI3 are significantly upregulated in SHH-MB, but GLI3 is not upregulated in the larger pool of medulloblastomas, and there is only weak evidence that GLI2 is upregulated. It is interesting to note that while GLI2 and GLI3 are upregulated, there is no significant difference in the detected number of $S H H$ transcripts. Additionally, PTKZ and EZH2 are significantly upregulated for both MB groups.

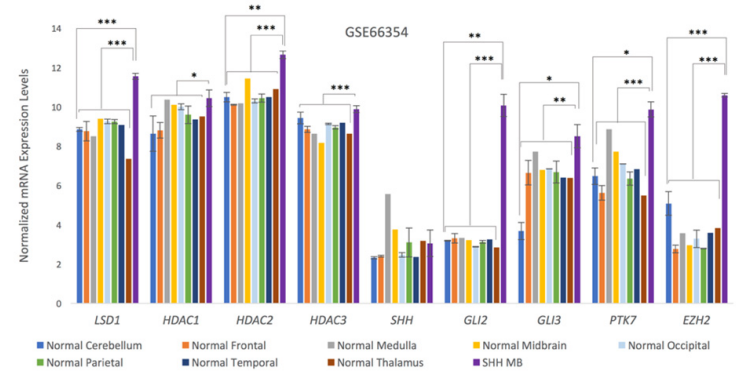

(A)

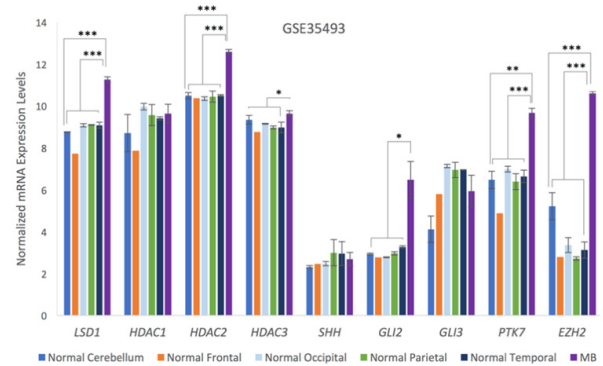

(B)

Figure 4. Normalized expression levels of key HDACs, SHH pathway genes, and other genes of interest in (A) human normal brain samples and SHH-MBs (GSE66354) based on microarray data from Griesinger et al. [11] or in (B) human normal brain samples and MBs of unspecified subtypes (GSE35493) based on microarray data from Birks et al. [12]. Error bars represent the standard error of the mean. Significance was tested across MBs ( $n=8$ GSE66354, $n=17$ GSE35493) versus normal cerebellum ( $n=2$ GSE66354, $n=2$ GSE35493) or all normal brain tissue ( $n=13$ GSE66354, $n=9$ GSE35493). $p$ values were adjusted using the Benjamini and Hochberg procedure [14]; $p<0.001\left(^{* * *}\right)$, $p<0.01\left(^{* *}\right), p<0.1\left(^{*}\right)$. 


\section{Discussion}

Currently, there are few effective therapies for pediatric medulloblastoma, with surgery often not able to remove residual metastastic tissue. In recent years, transcriptome profiling of human cancers has revealed important insights into the biology of tumors. In this brief report, following an analysis of microarrays of medulloblastoma patients, we identify key proteins-such as LSD1, EZH2, GLI2, GLI3, and PTK7 - that may serve as drug targets. In addition, we examine the efficacy of 4SC-202 in a pediatric medulloblastoma cell line. Our studies indicate that exposure of the medulloblastoma cells to 4SC-202 significantly reduces cell viability and provides the groundwork for a future more in-depth analysis of the effect of 4SC-202 on medulloblastoma, both in vitro and in preclinical models.

Upregulation of target proteins identified by analysis of the microarray data may be important targets for future therapies for medulloblastoma. As we have previously demonstrated, upregulation of PTK7 may indicate that it is an important target for pediatric cancers [3]. LSD1, HDAC2, and HDAC3 are key targets for 4SC-202, and thus upregulation of these targets across the studied medulloblastomas makes 4SC-202 a more actionable therapeutic.

GLI2 and GLI3 are two of the principle mediators of the sonic hedgehog signaling pathway (Shh) [15] and are upregulated in SHH-medulloblastomas (SHH-MB) relative to normal brain samples. It was recently demonstrated that selectively inhibiting HDAC1 and HDAC2 chemically decreases the tumor growth of SHH-MB in a murine model by inhibiting the Hedgehog pathway (Hh), which is further linked to an increase in GLI1 acetylation [16]. Likewise, the tumor killing activity of 4SC-202 may be related to the inhibition of the Hh pathway. Alternately, in pancreatic ductal adenocarcinoma (PDAC), it was found that 4SC-202 decreases TGF $\beta$ signaling [17]. TGF $\beta$ signaling and Hh signaling converge around GLI2 [18], and differences in the level of TGF $\beta$ signaling have been associated with slowed MB progression and improved patient outcomes, though the mechanism remains unclear [19-21]. Further preclinical studies and pathway studies need to be conducted to validate the efficacy and mechanism of action of 4SC-202 for in vivo models.

Acknowledgments: The authors would like to thank Erin Harmon for assistance in imaging the DAOY cells. This work was supported by Sanford Children's Health Dept., the BioSNTR grant UP1700139A, the National Science Foundation/EPSCoR Grant (IIA-1355423), an Institutional Development Award (IDeA) from the National Institute of General Medical Sciences of the National Institutes of Health (P20GM103443), and the Sanford Research Imaging Core, which is supported by the National Institutes of Health COBRE grants (P20 GM103620 and P20 GM103548).

Author Contributions: R.B. and S.M.M. conceived and designed the experiments; S.M.M. performed the experiments. M.M.H. and S.M.M. analyzed the data; E.Z.G. contributed to bioinformatics analysis. S.M.M. and M.H. wrote the paper. H.K. contributed to experiment design, data discussion and interpretation, and paper preparation.

Conflicts of Interest: H.K. was an employee of 4SC AG until 31 December, 2016. H.K. is currently affiliated with Immunic AG. 4SC-202 is still the property of 4SC AG and clinical trials are ongoing. H.K. holds a minor amount of shares in 4SC AG. Other authors claim no conflict of interest.

\section{References}

1. Reith, W.; Bodea, S.; Mühl-Benninghaus, R. Pediatric brain tumors. Radiologe 2017. [CrossRef]

2. Chakravadhanula, M.; Hampton, C.N.; Chodavadia, P.; Ozols, V.; Zhou, L.; Catchpoole, D.; Xu, J.; Erdreich-Epstein, A.; Bhardwaj, R.D. Wnt pathway in atypical teratoid rhabdoid tumors. Neuro. Oncol. 2014, 17, 526-535. [CrossRef] [PubMed]

3. Messerli, S.M.; Hoffman, M.M.; Gnimpieba, E.Z.; Bhardwaj, R.D. Therapeutic Targeting of PTK7 is Cytotoxic in Atypical Teratoid Rhabdoid Tumors. Mol. Cancer Res. 2017, 15, 973-983. [CrossRef] [PubMed]

4. Staedtke, V.; Dzaye, O.D.A.; Holdhoff, M. Actionable Molecular Biomarkers in Primary Brain Tumors. Trends Cancer 2016, 2, 338-349. [CrossRef] [PubMed]

5. Kohlhof, H.; Gruber, W.; Vitt, D.; Aberger, F.; Prenzel, T. Abstract C89: The small molecule inhibitor 4SC-202 controls aberrant HH signaling in cancer. Mol. Cancer Ther. 2015, 14. [CrossRef] 
6. Pinkerneil, M.; Hoffmann, M.J.; Kohlhof, H.; Schulz, W.A.; Niegisch, G. Evaluation of the Therapeutic Potential of the Novel Isotype Specific HDAC Inhibitor 4SC-202 in Urothelial Carcinoma Cell Lines. Target. Oncol. 2016, 11, 783-798. [CrossRef] [PubMed]

7. Henning, S.W.; Doblhofer, R.; Kohlhof, H.; Jankowsky, R.; Maier, T.; Beckers, T.; Schmidt, M.; Hentsch, B. Preclinical characterization of 4SC-202, a novel isotype specific HDAC inhibitor. EJC Suppl. 2010, 8, 61. [CrossRef]

8. Huang, Z.; Wang, S.; Min, H.; Li, J.; Qin, L.; Li, D. Pre-clinical characterization of 4SC-202, a novel class I HDAC inhibitor, against colorectal cancer cells. Tumor Biol. 2016, 37, 10257-10267.

9. Von Tresckow, B.; Gundermann, S.; Eichenauer, D.A.; Aulitzky, W.E.; Göbeler, M.; Sayehli, C.; Bacchus, L.; Hauns, B.; Mais, A.; Hentsch, B.; et al. First-in-human study of 4SC-202, a novel oral HDAC inhibitor in advanced hematologic malignancies (TOPAS study). J. Clin. Oncol. 2014, 32, 8559.

10. Elia, N.; Lippincott-Schwartz, J. Culturing MDCK cells in three dimensions for analyzing intracellular dynamics. Curr. Protoc. Cell Biol. 2009, 43, 1-18.

11. Griesinger, A.M.; Josephson, R.J.; Donson, A.M.; Mulcahy Levy, J.M.; Amani, V.; Birks, D.K.; Hoffman, L.M.; Furtek, S.L.; Reigan, P.; Handler, M.H.; et al. Interleukin-6/STAT3 Pathway Signaling Drives an Inflammatory Phenotype in Group A Ependymoma. Cancer Immunol. Res. 2015, 3, 1165-1174. [CrossRef] [PubMed]

12. Birks, D.K.; Donson, A.M.; Patel, P.R.; Sufit, A.; Algar, E.M.; Dunham, C.; Kleinschmidt-Demasters, B.K.; Handler, M.H.; Vibhakar, R.; Foreman, N.K. Pediatric rhabdoid tumors of kidney and brain show many differences in gene expression but share dysregulation of cell cycle and epigenetic effector genes. Pediatr. Blood Cancer 2013, 60, 1095-1102. [CrossRef] [PubMed]

13. Smyth, G.K. Limma: Linear models for microarray data. In Bioinformatics and Computational Biology Solutions Using R and Bioconductor; Gentleman, R.C., Carey, V., Dudoit, S., Irizarry, R., Huber, W., Eds.; Springer: New York, NY, USA, 2005; pp. 397-420.

14. Benjamini, Y.; Hochberg, Y. Controlling the false discovery rate: A practical and powerful approach to multiple testing. J. R. Stat. Soc. B 1995, 57, 289-300.

15. Pan, Y.; Bai, C.B.; Joyner, A.L.; Wang, B. Sonic hedgehog Signaling Regulates Gli2 Transcriptional Activity by Suppressing Its Processing and Degradation Sonic hedgehog Signaling Regulates Gli2 Transcriptional Activity by Suppressing Its Processing and Degradation. Mol. Cell. Biol. 2006, 26, 3365-3377. [CrossRef] [PubMed]

16. Coni, S.; Mancuso, A.B.; Di Magno, L.; Sdruscia, G.; Manni, S.; Serrao, S.M.; Rotili, D.; Spiombi, E.; Bufalieri, F.; Petroni, M.; et al. Selective targeting of HDAC1/2 elicits anticancer effects through Gli1 acetylation in preclinical models of SHH Medulloblastoma. Sci. Rep. 2017, 7, 44079. [CrossRef] [PubMed]

17. Mishra, V.K.; Wegwitz, F.; Kosinsky, R.L.; Sen, M.; Baumgartner, R.; Wulff, T.; Siveke, J.T.; Schildhaus, H.-U.; Najafova, Z.; Kari, V. Histone deacetylase class-I inhibition promotes epithelial gene expression in pancreatic cancer cells in a BRD4-and MYC-dependent manner. Nucleic Acids Res. 2017, 45, 6334-6349. [CrossRef] [PubMed]

18. Javelaud, D.; Alexaki, V.I.; Dennler, S.; Mohammad, K.S.; Guise, T.A.; Mauviel, A. TGF- $\beta /$ SMAD/GLI2 signaling axis in cancer progression and metastasis. Cancer Res. 2011, 71, 5606-5610. [CrossRef] [PubMed]

19. Aref, D.; Moffatt, C.J.; Agnihotri, S.; Ramaswamy, V.; Dubuc, A.M.; Northcott, P.A.; Taylor, M.D.; Perry, A.; Olson, J.M.; Eberhart, C.G.; et al. Canonical TGF- $\beta$ pathway activity is a predictor of SHH-driven medulloblastoma survival and delineates putative precursors in cerebellar development. Brain Pathol. 2013, 23, 178-191. [CrossRef] [PubMed]

20. Gate, D.; Danielpour, M.; Rodriguez, J.; Kim, G.-B.; Levy, R.; Bannykh, S.; Breunig, J.J.; Kaech, S.M.; Flavell, R.A.; Town, T. T-cell TGF-signaling abrogation restricts medulloblastoma progression. Proc. Natl. Acad. Sci. USA 2014, 111, E3458-E3466. [CrossRef] [PubMed]

21. Huang, S.Y.; Yang, J.Y. Targeting the hedgehog pathway in pediatric medulloblastoma. Cancers 2015, 7, 2110-2123. [CrossRef] [PubMed]

(c) 2017 by the authors. Licensee MDPI, Basel, Switzerland. This article is an open access article distributed under the terms and conditions of the Creative Commons Attribution (CC BY) license (http:/ / creativecommons.org/licenses/by/4.0/). 